\title{
Novel strategy for treatment of cancer cachexia based on metabolic alteration in central nervous system in a mouse model
}

\author{
Miaki Uzu', Miki Nonaka', Kanako Miyano ${ }^{1}$, Hiromi Sato ${ }^{2}$, Nagomi Kurebayashi ${ }^{3}$, \\ Takashi Murayama ${ }^{3}$, Takashi Sakurai ${ }^{3}$, Akihiro Hisaka ${ }^{2}$, Yasuhito Uezono ${ }^{1,4}$ \\ ${ }^{I}$ Division of Cancer Pathophysiology, National Cancer Center Research Institute, Japan, ${ }^{2}$ Laboratory of Clinical \\ Pharmacology and Pharmacometrics, Graduate School of Pharmaceutical Sciences, Chiba University, Japan, \\ ${ }^{3}$ Department of Pharmacology, Juntendo University School of Medicine, Japan, ${ }^{4}$ Division of Supportive Care Research, \\ Exploratory Oncology Research and Clinical Trial Center, National Cancer Center Research Institute, Japan
}

[Background] Cancer cachexia is a systemic wasting syndrome, which is characterized by anorexia and the loss of body weight, adipose tissue, and skeletal muscle. This syndrome causes poor quality of life and poor responses to chemotherapy in advanced cancer patients, thus, therapeutic strategies for cancer cachexia need to be established. However, the lack of appropriate animal models has resulted in the poor understanding of the mechanisms causing and developing cachexic symptoms. We thus have established a novel animal model, which meets clinical criteria for cachexia (Terawaki K. et al., 2014). In this study, by using this model, metabolic changes in central nervous system (CNS), which may control an appetite, from early to late stages of cachexia was assessed, and the efficacy of targeting the altered metabolic pathway in the CNS was investigated.

[Methods] Anesthetized 8-week-old male BALB/c nu/nu mice were subcutaneously inoculated with a human gastric cancer cell line, 85As2. Body weight, tumor volume, food and water consumption were evaluated weekly. Two or six weeks later, the mice were sacrificed and organs (brain, heart, lung, skeletal muscle, and epididymal fat) were weighed. Quantitative alteration of approximately 90 kinds of metabolites (neurotransmitters, nucleic acids, amino acids, and intermediates in glucose metabolism) in the brain was determined using a capillary electrophoresis-time of flight mass spectrometry (CE-TOFMS) system.

[Results] Subcutaneous implantation of 85As2 cells induced progressive tumor growth and significant body weight loss was observed in two weeks, accompanied by gradual decrease of food and water consumption. Metabolome analysis using brains collected two weeks after implantation showed lower amounts of purine nucleotides (IMP, AMP, ADP, ATP, GMP, GDP, and GTP) in the brains of 85As2-induced cancer cachexia mice than in those of control group (Fig.1). On the other hand, quantification of the downstream metabolites revealed that the amounts of inosine and hypoxanthine were higher in the brains of 85As2-induced cancer cachexia mice.

[Conclusion] These results suggest that the purine nucleotide metabolism is activated at the onset of cancer cachexia, which might be a novel therapeutic target for the treatment of this syndrome. Therefore, the effect of agents targeting this pathway is being investigated. 